\title{
Plasmonic-Enhanced Organic Light-Emitting Diodes Based on a Graphene Oxide/Au Nanoparticles Composite Hole Injection Layer
}

\author{
Jiahuan Feng ${ }^{1,2 t}$, Dongwei Sun ${ }^{1,2 t}$, Sijiong Mei ${ }^{1,2}$, Wuxing Shi ${ }^{1,2}$, Fei Mei ${ }^{1,2 *}$, \\ Yuanming Zhou ${ }^{1,2 *}$, Jinxia $\mathrm{Xu}^{1,2}$, Yan Jiang ${ }^{1,2}$ and Linzhang $\mathrm{Wu}^{2}$ \\ ${ }^{1}$ Hubei Key Laboratory for High-efficiency Utilization of Solar Energy and Operation Control of Energy Storage System, Hubei \\ University of Technology, Wuhan, China, ${ }^{2}$ School of Electrical and Electronic Engineering, Hubei University of Technology, \\ Wuhan, China
}

OPEN ACCESS

Edited by:

Xiangheng Xiao,

Wuhan University, China

Reviewed by:

Wu Wei,

Wuhan University, China

Guolin Yu,

Shanghai Institute of Technical

Physics (CAS), China

Wei Bai,

East China Normal University, China

*Correspondence:

Fei Mei

meifei777@163.com

Yuanming Zhou

zhouym@mail.hbut.edu.cn

${ }^{\dagger}$ These authors have contributed equally to this work

Specialty section:

This article was submitted to Translational Materials Science,

a section of the journal

Frontiers in Materials

Received: 31 October 2018 Accepted: 28 November 2018 Published: 13 December 2018

Citation:

Feng J, Sun D, Mei S, Shi W, Mei F Zhou Y, Xu J, Jiang Y and Wu L (2018)

Plasmonic-Enhanced Organic

Light-Emitting Diodes Based on a

Graphene Oxide/Au Nanoparticles Composite Hole Injection Layer.

Front. Mater. 5:75

doi: 10.3389/fmats.2018.00075
Organic light-emitting diodes (OLEDs) have drawn a great deal of attention due to their broad applications in lighting and displaying. With the development of nanotechnology, surface plasmas have been widely used in photonics, microscopes, solar cells and biosensors. In this paper, by inserting graphene oxide (GO), Au nanoparticles (Au NPs) and GO/Au NP composite structures between the hole transport layer (NPB) and indium tin oxide (ITO) anode, respectively, the electroluminescent performance of $\mathrm{Alq}_{3}$-based OLEDs was significantly enhanced. Compared to the reference devices, the devices with the composite inserting layer containing 10\% GO/Au NP doping have the best electroluminescent performance, which improved $47.9 \%$ in maximum luminance, $49.2 \%$ in maximum current efficiency and $45.3 \%$ in maximum external quantum efficiency (EQE). Such substantial enhancement of photoelectric performance can be attributed to the combined effects of LSPR coupling and the better hole transport property by introducing Au NPs and a graphene oxide-doped layer.

Keywords: organic light-emitting diodes (OLED), surface plasmon resonance (SPR), graphene oxide (GO), Au nanoparticles (Au NPs), hole transport layer (HTL)

\section{INTRODUCTION}

Organic light-emitting diodes (OLEDs) have drawn a great deal of attention due to their broad applications in lighting and displaying because they are simple to produce, lightweight, and flexible (Park et al., 2017). The improved efficiency and enhanced device stability are important for their commercialization (Coehoorn et al., 2015; Zhang et al., 2015b) Tremendous effort has been made by many researchers to enhance electroluminescent performance of OLEDs in different ways (Jesuraj et al., 2017). These efforts include heightening the internal quantum efficiency of organic materials (Adachi et al., 2001; Xiao et al., 2009), improving the out-coupling efficiency of the device with an array of lenses (Sun and Forrest, 2008; Han et al., 2018), metal nanoparticles (NPs) (Chen et al., 2017; Zhang et al., 2017), and reasonable nanostructures (Riedel et al., 2010; Chen et al., 2015).

Carbon-based 2-dimensional (2-D) materials such as graphene and graphene oxide (GO) have been widely used in optoelectronic devices due to their unique physical and electronic properties (Wu et al., 2013, 2014b; Basu et al., 2014; Kim et al., 2014; Yeo et al., 2015). Graphene and graphene oxide have great potential for large-area electronics because of their large surface area, 
high intrinsic mobility, high optical transmittance, etc (GómezNavarro et al., 2007; Zhu et al., 2010; Chen et al., 2011; Chabot et al., 2014; Naumov et al., 2016; Jesuraj et al., 2017). The existence of oxygen functional groups on the GO structure makes it dispersible in many solvents and enables its utilization in solution-processable devices (Yun et al., 2011; Shi et al., 2013; Liang et al., 2014; Lee et al., 2015, 2016).

Another effective method, namely the use of plasmonic metallic nanoparticles, has also been used to improve the electroluminescent properties of OLEDs (Choi et al., 2013; Wu et al., 2014a, 2018; Zhang et al., 2015a, 2017; Cho et al., 2016; Balakrishnan and Kamat, 2017). One of the most important properties of plasmonic metallic nanoparticles is surface plasmon resonance (SPR), which is the strong interaction between plasmonic metallic nanoparticles and resonant photons. SPR could promote light emission effectively through an enhanced spontaneous emission rate and thus improve the performance of OLEDs (Xiao et al., 2012; Xu et al., 2017). Furthermore, the light scattering caused by the plasmonic metallic nanoparticles can help extract photons trapped within the substrate or waveguide modes, which increases light extraction efficiency (Chen et al., 2017). The prerequisite to strengthening the coupling effect between metallic nanoparticles and resonant photons is the overlapping between the photoluminescence (PL) spectrum emission layer and the absorption spectrum of metallic nanoparticles, which can be modified by the morphology of the metallic nanoparticles we selected.

As presented in the literature, the electroluminescent performance of OLEDs has been improved by introducing a 2D material between the anode and the hole transport layer (HTL) or utilizing the surface plasmon resonance (SPR) of metallic nanoparticles (MNPs) individually. However, the combination of these two effects has rarely been reported in the research field of OLEDs. In this work, a composite structure comprising $\mathrm{Au}$ NPs embedded in GO was introduced as a hole injection layer to take advantage of $2 \mathrm{D}$ materials and the plasma effect, which enhance the electroluminescence performance of OLEDs. The volume ratio of Au NPs and the concentration of GO were changed to investigate the optical and electrical properties of the OLED devices. The optimal values of the volume ratio of Au NPs and the GO concentration were found to be $10 \%$ and $0.25 \mathrm{mg} / \mathrm{ml}$, respectively. Correspondingly, the OLEDs with GO/Au NPs prepared under these conditions also have the best performance. Compared with the reference devices without Au NPs and GO, the optimal plasmonic OLEDs exhibit better performance in terms of luminance, current efficiency, and EQE. These results suggest that the plasmonic OLEDs have great potential for enhanced performance by introducing Au NPs and GO.

\section{THE EXPERIMENT}

\section{Materials Used}

All solvents and reagents were used as received unless otherwise stated. Tetrachloroauratetrihydrate $\left(\mathrm{HAuCl}_{4} \cdot 3 \mathrm{H}_{2} \mathrm{O}, 99.99 \%\right)$, Trisodium citrate dihydrate $\left(\mathrm{Na}_{3} \mathrm{C}_{6} \mathrm{H}_{5} \mathrm{O}_{7} \cdot 2 \mathrm{H}_{2} \mathrm{O}\right.$, 99.0\%) and n-butylamine $\left(\mathrm{C}_{4} \mathrm{H}_{11} \mathrm{~N}, 99.0 \%\right)$ were purchased from Shanghai Chemical Reagent Co, Ltd (China). N, N'-Bis
(naphthalen-1-yl)-N, N'-bis (diphenyl) benzidine (NPB, 99.5\%) and Tris-(8-Hydroxyquinolinato) aluminum ( $\left.\mathrm{Alq}_{3}, 99.5 \%\right)$ were purchased from Jilin Optical and Electronic Materials Co, LTD (China). Lithium Fluoride (LiF, 99.995\%) and Aluminum slug (Al, 99.999\%) were purchased from Sigma Aldrich and Alfa Aesar, respectively.

\section{Synthesis of Graphene Oxide and Gold Nanoparticles}

First, the GO solution was prepared from natural graphite powder by a modified Hummers method. Then, the obtained GO solution was purified through centrifugation $(12,000 \mathrm{rpm}$ for $60 \mathrm{~min}$ ) and washed with deionized water several times until the GO solution was neutral, with a $\mathrm{pH}$ value of 7 . Through these steps, we finally got the GO aqueous solution with a concentration of $1 \mathrm{mg} / \mathrm{ml}$. Before being used in the fabrication of OLEDs, the GO solution would be stirred through ultrasonication for more than $1 \mathrm{~h}$.

$\mathrm{Au}$ NPs with $20 \mathrm{~nm}$ diameter were synthesized according to Frens method. The synthesis procedure of Au NPs is based on the $\mathrm{HAuCl}_{4}$ solution and trisodium citrate dihydrate as a reducing agent. Briefly, Au NPs $20 \mathrm{~nm}$ in diameter were prepared by adding aqueous $\mathrm{HAuCl}_{4}(0.5 \mathrm{ml}, 0.05 \mathrm{M})$ into deionized water $(19.5 \mathrm{ml})$ in a glass tube, and then the obtained solution was brought to a boil while being vigorously stirred. Then, $1.8 \mathrm{ml}$ $0.05 \mathrm{~g} / \mathrm{ml}$ aqueous trisodium citrate dihydrate was added to the solution. The reaction solution was kept at a boil for $10 \mathrm{~min}$ until the solution's color was wine red, indicating that the Au NPs with desired size had been synthesized.

The synthesis process of GO/Au NPs composites was followed by an earlier reported procedure (Fu et al., 2016). $0.25 \mathrm{ml} \mathrm{n-}$ butylamine was added to the obtained GO $(5 \mathrm{ml} 1.0 \mathrm{mg} / \mathrm{ml})$ and then the mixed solution was stirred for $45 \mathrm{~min}$, forming a uniform GO colloid. Then, $5 \mathrm{ml}$ of synthesized solution containing Au NPs was added to the GO colloid. Subsequently, the mixture was heated to $80^{\circ} \mathrm{C}$ with magnetic stirring and aged at the same temperature overnight. The GO/Au NPs were washed several times and re-dispersed in $5 \mathrm{ml}$ of deionized water before use.

\section{OLED Fabrication}

The OLEDs were fabricated on the precleaned patterned ITO glass substrates, whose sheet resistance was $\sim 15 \Omega / \mathrm{m}^{2}$. The ITO substrates were ultrasonically cleaned with acetone, ethanol and deionized water in sequence for $10 \mathrm{~min}$ respectively, and then treated with oxygen plasma for $10 \mathrm{~min}$ to increase the work function. After these steps, different hole injection layers (HILs), such as GO, Au NPs, and GO/Au NPs, were spin-coated on ITO substrates at 6,000 rmp for $45 \mathrm{~s}$ and then annealed at $80^{\circ} \mathrm{C}$ for $10 \mathrm{~min}$. The typical OLED structure consists of a HIL (GO, Au NPs, or GO/Au NPs composite), a $30 \mathrm{~nm} \mathrm{NPB}$ as HTL, a $30 \mathrm{~nm}$ $\mathrm{Alq}_{3}$ as an emitting layer (EML), a $30 \mathrm{~nm} \mathrm{Alq}$ as an electron transport layer (ETL), a $0.5 \mathrm{~nm} \mathrm{LiF}$ as an electron inject layer (EIL) and $100 \mathrm{~nm} \mathrm{Al}$ as a cathode deposited through a special mask, which defines the active area of all these devices to be 0.1 $\mathrm{cm}^{2}$. Four identical devices on each substrate were encapsulated with a glass lid in a $\mathrm{N}_{2}$-filled glove box and then characterized at room temperature. 
A

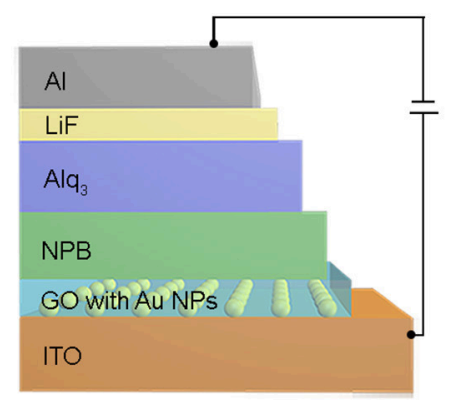

C

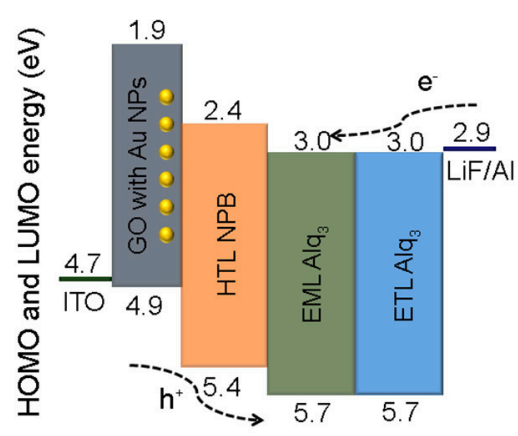

B
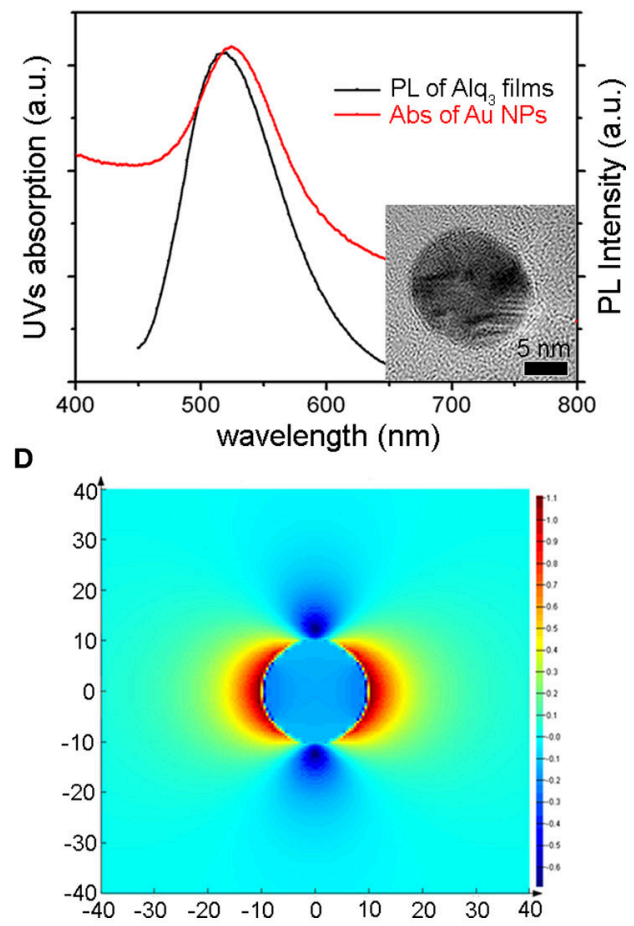

FIGURE 1 | (A) The device structure of the OLEDs by inserting GO/Au NPs between the ITO anode and NPB hole transport layer. (B) The absorption spectrum of Au NP solution and the PL spectrum of $\mathrm{Alq}_{3}$ film on ITO glass. The inset shows the TEM image of the Au NPs. (C) The energy level diagram of the OLEDs. (D) FDTD stimulation of the electromagnetic field distribution around Au NPs with the diameter of $20 \mathrm{~nm}$.

\section{Characterization}

The current density-voltage (J-V) characteristics of the devices were tested using a Keithley 2400 source measure unit, and the EL spectra of the OLEDs were recorded using an Ocean Optics fiber-optic spectrometer. The luminance of the devices was measured by a Keithley 2000 multimeter coupled with a calibrated silicon photodetector $(1 \mathrm{~cm}$ in diameter), which was put directly onto the surface of an individual device, ensuring that all photons emitted from the glass side were captured. In this procedure, the operating current density is set to be 50 $\mathrm{mA} / \mathrm{cm}^{2}$.

The photoluminescence (PL) absorption spectra were measured by a HITACHI F-4600 luminescence spectrometer with an excitation wavelength of $430 \mathrm{~nm}$, and the UV-vis spectra were monitored with a HITACHI U-3900 ultraviolet/visible spectrophotometer. The surface morphology was conducted via scanning electron microscopy (SEM, FEI Sirion FEG). Transmission electron microscopy (TEM, FEI TECNAI G2 20) was used to characterize the topography of Au NPs. The finite-difference timed-domain (FDTD) simulations have been performed to reveal the Au NP-induced plasmon modes.

\section{RESULTS AND DISCUSSION}

The typical device configuration consists of spin-coated layers of the GO/Au NPs on the surface of the ITO anode and evaporated layers of $\mathrm{NPB}, \mathrm{Alq}_{3}$ and $\mathrm{LiF} / \mathrm{Al}$, as shown in the schematic diagram in Figure 1A. GO/Au NPs were capped by NPB via spin-coating its aqueous solution on the ITO anode. Figure 1B shows the optical properties of the Au NPs and the evaporated $\mathrm{Alq}_{3}$ film. The TEM image indicates that the $\mathrm{Au}$ NPs are spherical in shape and the average diameter is nearly $20 \mathrm{~nm}$. The Au NPs display a typical absorption peak of $524 \mathrm{~nm}$, which is seen in the UV-vis absorption spectrum, while the PL spectrum of the $\mathrm{Alq}_{3}$ film has a peak position around $520 \mathrm{~nm}$. It is evident that the absorption peak of the Au NPs matches the PL peak of the $\mathrm{Alq}_{3}$ films. The overlap of these two spectra provides the possibility for resonance between radiated light generated in the $\mathrm{Alq}_{3}$ emitter and localized SPR excited by the $\mathrm{Au}$ NPs, which is expected to result further in effective energy transfer and, correspondingly, enhanced emission intensity (Zhang et al., 2017).

The energy level values in Figure 1C were taken from Ref (Liu et al., 2017) and ( $\mathrm{Li}$ et al., 2017). It can be seen that the injection of holes from ITO into the $\mathrm{Alq}_{3}$ layer was partly prohibited due to the large energy barrier of $\sim 0.9-1.0 \mathrm{eV}$, while electrons can be easily injected into $\mathrm{Alq}_{3}$ from $\mathrm{LiF} / \mathrm{Al}$. The ITO glass was modified with GO-a material with a high work function (WF) - in order to enhance the hole injection efficiency, finally achieving more balanced hole and electron transport. Figure 1D shows the FDTD stimulation of the electromagnetic field distribution around Au NPs with a diameter of $20 \mathrm{~nm}$, which indicates that the effective range of LSPR is $\sim 10 \mathrm{~nm}$. According 

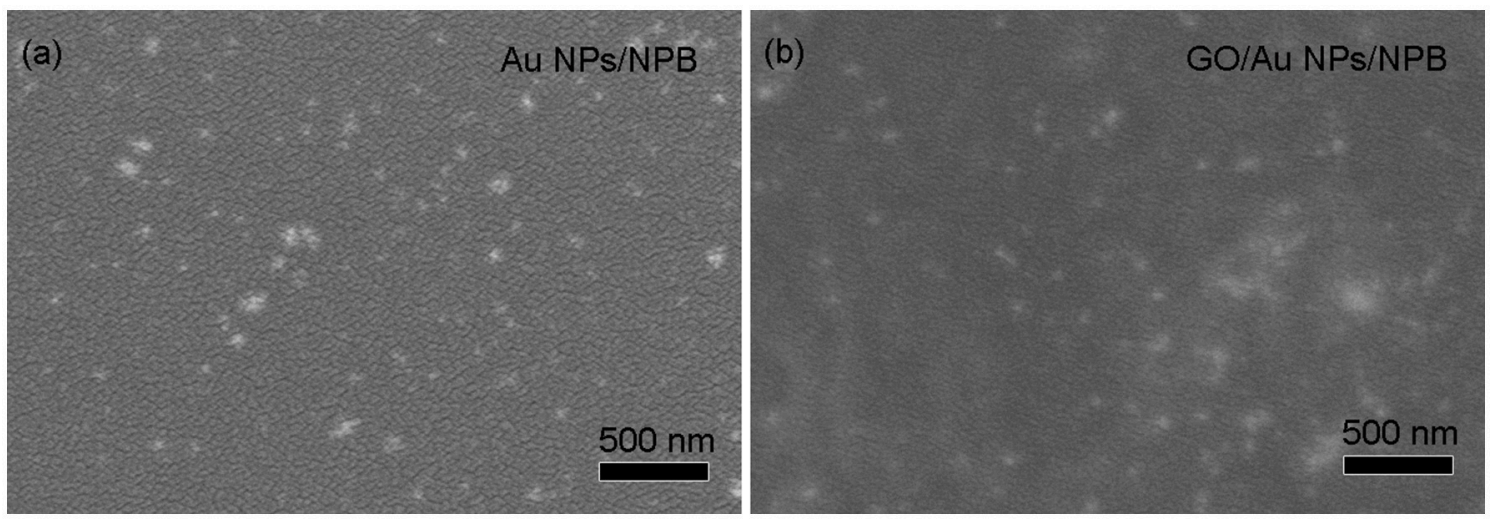

FIGURE 2 | (A) The SEM image of NPB film with Au NPs, in which the volume ratio of Au NPs is 10\%. (B) The SEM image of NPB film with GO/Au NPs, in which the volume ratio of $\mathrm{GO} / \mathrm{Au} \mathrm{NPs}$ is $10 \%$.
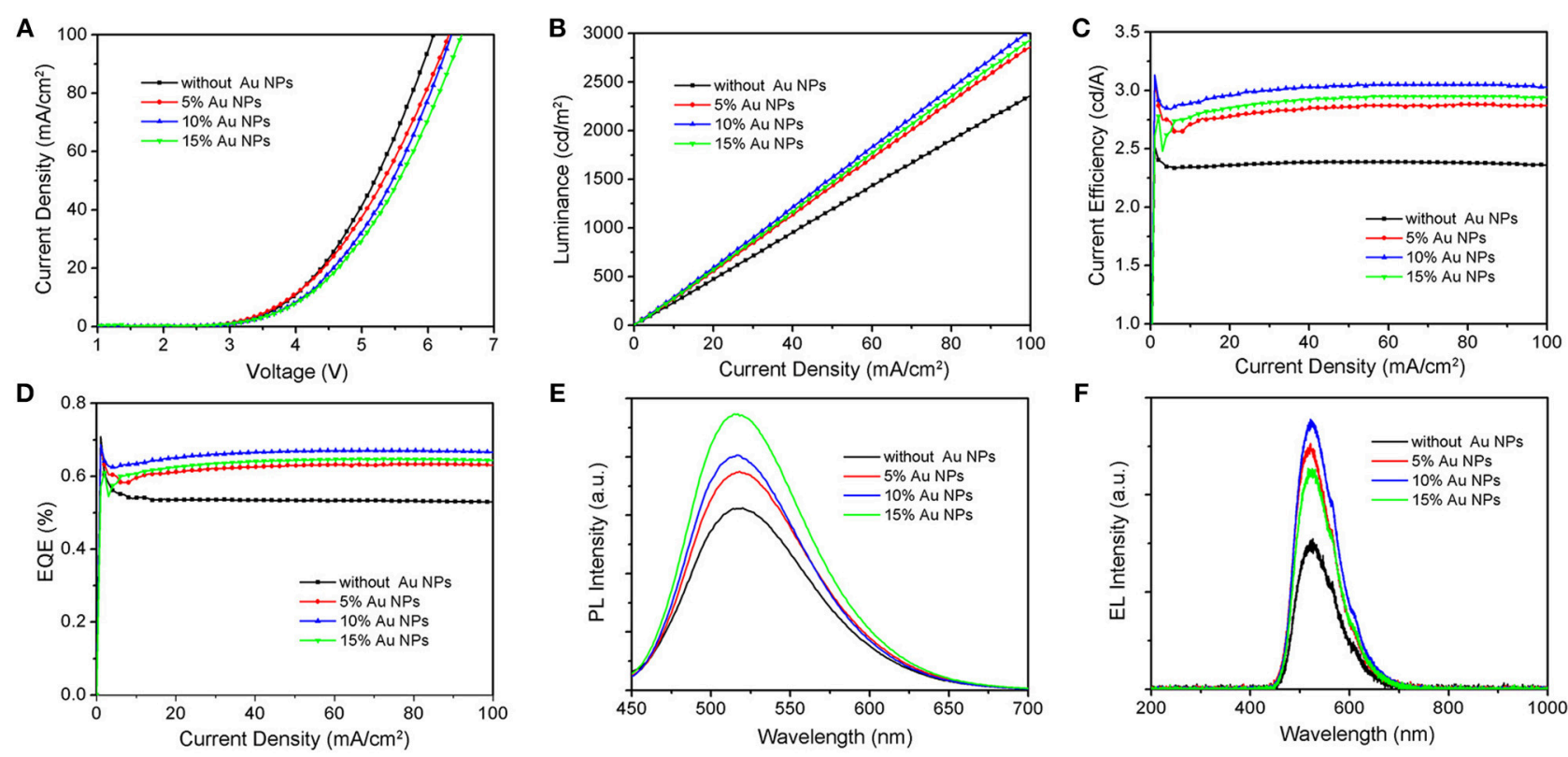

$\mathbf{E}$

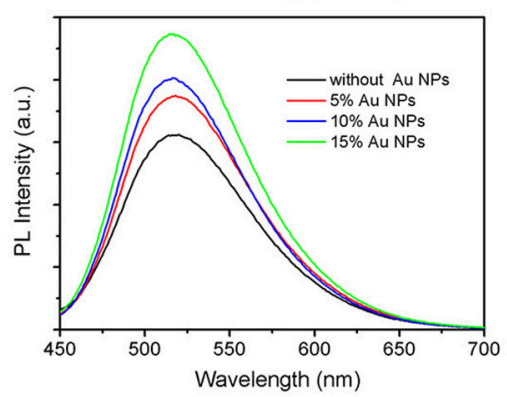

$\mathbf{F}$

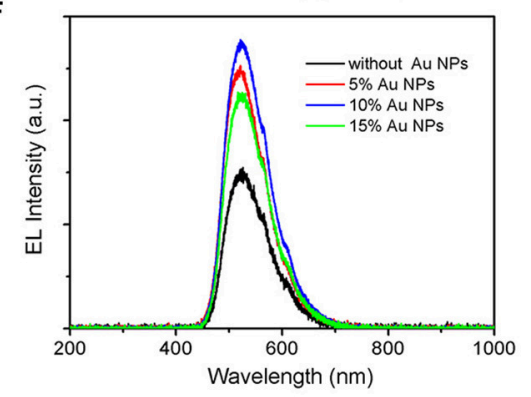

FIGURE 3 | Characteristics of OLEDs with only Au NPs. (A) The J-V characteristics, (B) L-J characteristics, (C) current efficiency, (D) EQE vs. current density characteristics of OLEDs with different volume ratios of Au NPs, (E) PL spectra of $\mathrm{Alq}_{3}$ film and (F) EL spectra of OLEDs with different volume ratios of Au NPs operated at $50 \mathrm{~mA} / \mathrm{cm}^{2}$.

to our device structure, the exciton recombination zone would be very close to the $\mathrm{NPB} / \mathrm{Alq}_{3}$ interface, which can fall into the electromagnetic fields of Au NPs.

In order to define the effect of $\mathrm{Au}$ NPs and GO/Au NP composites on the surface morphology of the NPB film, we studied the SEM images of the NPB films, which are located on the $\mathrm{Au}$ NPs and GO/Au NPs composite, respectively. The SEM images showed that the NPB films' morphology changes with $\mathrm{Au}$ NPs and GO/Au NPs in Figures 2A,B, respectively. Several Au NPs seen as white spots are distributed randomly in the dense and uniform background, which is the typical image the NPB film fabricated by thermal evaporation. It was expected that introducing $\mathrm{GO}$ and $\mathrm{Au}$ NPs would not cause notable morphology changes to the NPB films.
The J-L-V characteristics of the OLEDs with different volume ratios of Au NPs are illustrated in Figure 3. According to the J-V curves in Figure 3A, on increasing the volume ratio of Au NPs, the current density of the OLED devices decreases at the same bias voltage. In contrast to the trend of J-V characteristics, two distinct evolutions of the maximum luminance were observed in the L-J curves. As the Au NPs' volume ratio increase from 0 to $10 \%$, the maximum luminance increases from $2,380 \mathrm{~cd} / \mathrm{m}^{2}$ to $3,050 \mathrm{~cd} / \mathrm{m}^{2}$, which is $28.2 \%$ higher than that of the control OLEDs. However, when the Au NPs' volume ratio is higher than $10 \%$, the maximum luminance decreases continuously with the Au NPs' volume ratio. The current efficiency and EQE characteristics of the OLEDs are shown in Figures 3C,D, respectively. Compared to the maximum current efficiency and EQE of OLEDs without Au NPs, significant enhancements of 28.2 
and $26.4 \%$ were obtained in the device with $10 \%$ Au NPs, which is very similar to the trend of L-J characteristics. A further increase of the volume ratio of Au NPs leads to a decrease of the current efficiency and EQE.
As shown in Figure 3E, the PL intensity continues to increase when the $\mathrm{Au}$ NPs' doping rate increased from 0 to $15 \%$, suggesting that the SPR effects were also enhanced with larger concentrations of Au NPs. However, the EL performance of
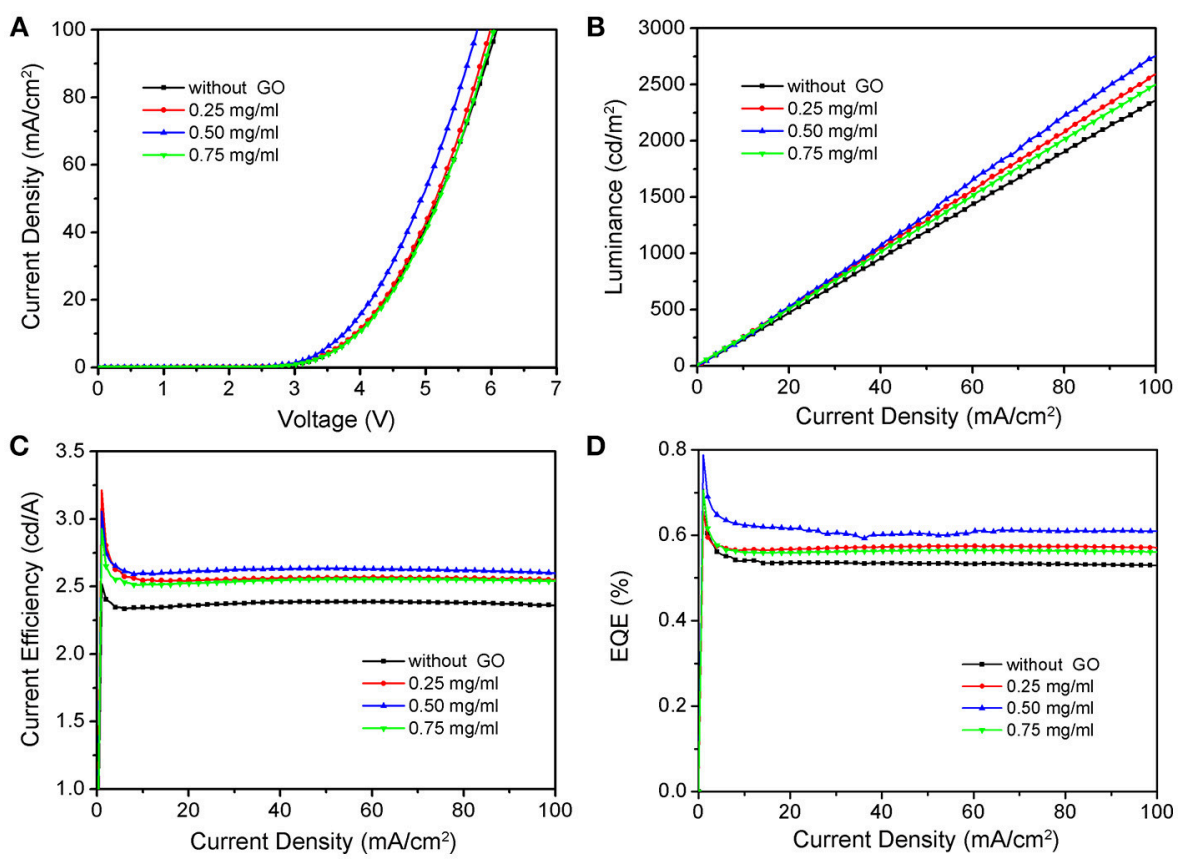

FIGURE 4 | Characteristics of OLEDs with only GO. (A) The J-V characteristics, (B) L-J characteristics, (C) current efficiency, and (D) EQE vs. current density characteristics of OLEDs with different concentrations of GO.
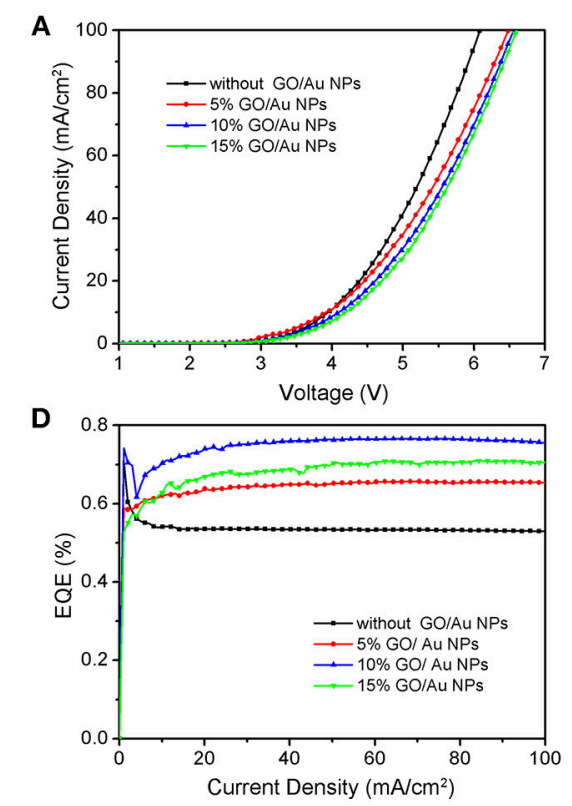

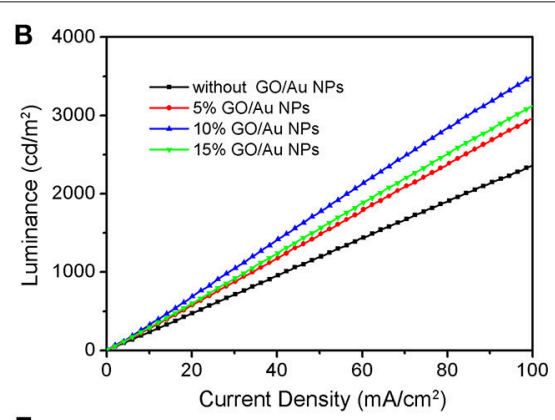

E

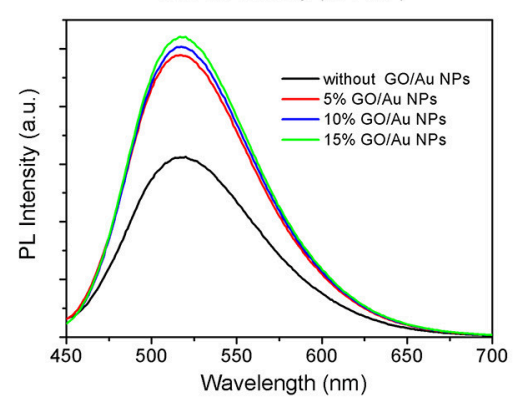

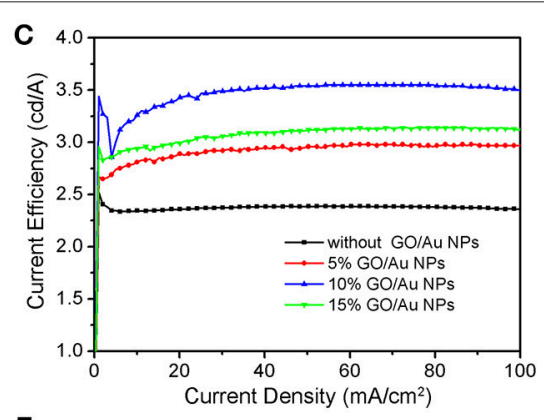

F

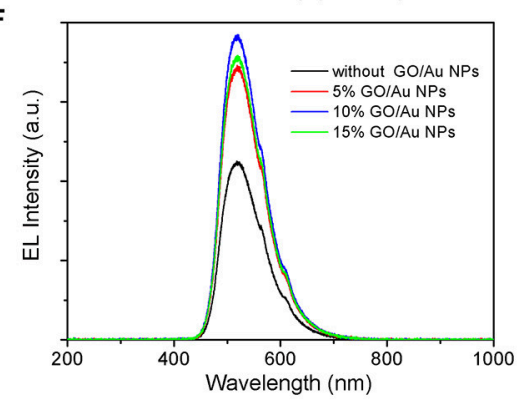

FIGURE 5 | Characteristics of OLEDs with GO/Au NPs. (A) The J-V characteristics, (B) L-J characteristics, (C) current efficiency, (D) EQE vs. current density characteristics of OLEDs with different volume ratios of GO/Au NPs and (E) PL spectra of Alq 3 film and (F) EL spectra of OLEDs with different volume ratios of GO/Au NPs operated at $50 \mathrm{~mA} / \mathrm{cm}^{2}$. 
the OLEDs, presented in Figure 3F, started to decrease while the $\mathrm{Au}$ NPs' volume ratios $>10 \%$, similar to the trend for maximum luminance. This can be caused by the competition between two effects, explained as follows: On the one hand, emission strength can be enhanced by the SPR effect caused by the Au NPs. On the other hand, the introduction of Au NPs with higher concentrations may destroy the flat film morphology, resulting in a slow decrease of the current density and poor electroluminescent performance of OLEDs (Chen et al., 2017). All the devices with Au NPs exhibit EL peaks around $520 \mathrm{~nm}$, which is consistent with the PL spectra of the $\mathrm{Alq}_{3}$ films.

The characteristics of the devices with GO interlayers are shown in Figure 4. In this work, GO with three low concentrations $(0.25,0.5$, and $0.75 \mathrm{mg} / \mathrm{ml})$ was investigated as a hole injection layer in OLEDs. Meanwhile, the transmittances of ITO and ITO/GO substrates are near-identical, which cannot impede the light emission of OLEDs. The devices with GO interlayers show better performance than the control device without GO in terms of turn-on voltage, luminance, current efficiency, and EQE. For example, the use of $0.5 \mathrm{mg} / \mathrm{ml} \mathrm{GO}$ leads to a decline of the turn-on voltage from 3.2 to $3.0 \mathrm{~V}$, an improvement of EQE from 0.53 to $0.62 \%$ and a significant enhancement of more than $16 \%$ in the maximum luminance.

The improvements in device performance can be attributed to the functionality of the GO interlayer. Because all the devices have the same structure on the cathode side, we deduce that the hole injection is improved by the GO interlayer. According to the proposed energy level diagram shown in Figure 1C, the large band gap of GO hinders the transport of electrons from the $\mathrm{Al}$ cathode to the ITO, acting as an effective electron blocking layer, which will improve electron-hole recombination in the active layer. Furthermore, the insertion of GO with an improved effective work function (WF) can induce an interface energy step between ITO and $\mathrm{Alq}_{3}$, leading to a reduced energy barrier and an improvement of hole injection efficiency with a concomitant increase in luminance.

In order to obtain the OLEDs with better performance, we also prepared a series of GO/Au NP composites, which were utilized as the hole injection layers for OLEDs. The properties of these devices were presented through the J-L-V characteristics in Figure 5. A substantial enhancement of electroluminescent performance was observed in the OLEDs with GO/Au NP composite structures and the optimal volume ratio was found to be $10 \%$. For the optimal OLEDs, the maximum luminance is $\sim 3,520 \mathrm{~cd} / \mathrm{m}^{2}$, which was $47.9 \%$ higher than that of the reference device without GO/Au NP composites. Very similar to the trend of J-L characteristics, the maximum current efficiency and EQE first increase with the volume ratio of GO/Au NPs, increasing from 0 to $10 \%$ and then decreasing with a further increase of the volume ratio. Additionally, both the maximum current efficiency of $\sim 3.55 \mathrm{~cd} / \mathrm{A}$ and the maximum EQE of $\sim 0.77 \%$ were achieved for the devices with $10 \% \mathrm{GO} / \mathrm{Au}$ NPs, which show substantial enhancements of 49.2 and $45.3 \%$, respectively. On increasing the volume ratio of GO/Au NPs to $15 \%$ or decreasing to 0 , both current efficiency and EQE decrease. The PL and EL spectra shown in Figures 5E,F are similar to those in Figure 3. Furthermore, it is noted that the increase of both PL and EL intensity is more evident after the insertion of GO. As explained above in Figure 3, despite the enhanced SPR effect, the excessive Au NPs may also cause serious morphological destruction of NPB film, which may take place in the devices with GO/Au NPs, also leading to the slight decrease of the current density.

It is reasonable to conclude that some effects, especially the LSPR coupling at the $\mathrm{NPB} / \mathrm{Alq}_{3}$ interface by introducing Au NPs and the improved hole injection by the GO interlayer, have taken place at the ITO/GO/Au NP/NPB interfaces, and help to improve the efficiency of $\mathrm{Alq}_{3}$ OLEDs. First, the resonance between radiated light generated in the $\mathrm{Alq}_{3}$ emitter and localized SPR excited by the Au NPs can lead to an effective energy transfer and, correspondingly, to enhanced emission intensity. Furthermore, GO can optimize the energy level structure of the device and balance electron and hole injection by blocking the flow of electrons from the emissive layer to the ITO and increasing the efficiency of the hole injection, thus further enhancing device performance of OLEDs (Silva et al., 2013).

\section{CONCLUSIONS}

In summary, we have demonstrated the enhanced luminescence of $\mathrm{Alq}_{3}$ OLEDs with Au NPs, GO and GO/Au NPs interlayer. Compared to the reference devices without GO or Au NPs, the OLEDs with GO/Au NPs exhibit the best performance, with a $47.9 \%$ enhancement in maximum luminance, a $49.2 \%$ enhancement in maximum current efficiency, and a $45.3 \%$ enhancement in EQE. These results can be explained by the SPR coupling between Au NPs and $\mathrm{Alq}_{3}$ excitons as well as the optimized band arrangement by inserting the GO interlayer. As discussed, SPR can facilitate effective energy transfer while the insertion of GO can create more balanced hole and electron transport, with both improving LED performance through enhanced radiative recombination rate. Our results illustrate that the combination between plasmonic Au NPs and GO is effective and has great potential in developing high-efficiency OLEDs, which provides a new way for improving OLED performance.

\section{AUTHOR CONTRIBUTIONS}

All authors listed have made a substantial, direct and intellectual contribution to the work, and approved it for publication.

\section{ACKNOWLEDGMENTS}

This work was supported by the Foundation of Hubei Provincial Science and Technology Department (Grant No. 2016BKJ005), the Leading Plan of Green Industry of Hubei University of Technology (Grant No. YXQN2016003), the International Science and Technology Cooperation Program of China (Grant No. 2016YFE0124300), the Open Foundation of Hubei Key Laboratory for High-efficiency Utilization of Solar Energy and Operation Control of Energy Storage System (Grant Nos. HBSEES201801, HBSEES201705), and the National Natural Science Foundation of China (Grant Nos. 51602099, 51371079, 11304092, and 11375134). 


\section{REFERENCES}

Adachi, C., Baldo, M. A., Thompson, M. E., and Forrest, S. R. (2001). Nearly $100 \%$ internal phosphorescence efficiency in an organic light emitting device. J. Appl. Phys. 90:5048. doi: 10.1063/1.1409582

Balakrishnan, S. K., and Kamat, P. V. (2017). Au-CsPbBr3 hybrid architecture. anchoring gold nanoparticles on cubic perovskite nanocrystals. ACS Energy Lett. 2, 88-93. doi: 10.1021/acsenergylett.6b00592

Basu, S., Lee, M. C., and Wang, Y. H. (2014). Graphene-based electrodes for enhanced organic thin film transistors based on pentacene. Phys. Chem. Chem. Phys. 16, 16701-16710. doi: 10.1039/C3CP55440F

Chabot, V., Higgins, D., Yu, A., Xiao, X., Chen, Z., and Zhang, J. (2014). A review of graphene and graphene oxide sponge: material synthesis and applications to energy and the environment. Energ. Environ. Sci. 7, 1564-1596. doi: 10.1039/C3EE43385D

Chen, C. Y., Lee, W. K., Chen, Y. J., Lu, C. Y., Lin, H. Y., and Wu, C. C. (2015). Enhancing optical out-coupling of organic light-emitting devices with Nanostructured composite electrodes consisting of indium tin oxide nanomesh and conducting polymer. Adv. Mater. 27, 4883-4888. doi: 10.1002/adma.201502516

Chen, P., Xiong, Z., Wu, X., Shao, M., Meng, Y., Xiong, Z. H., et al. (2017). Nearly $100 \%$ efficiency enhancement of $\mathrm{CH} 3 \mathrm{NH} 3 \mathrm{PbBr} 3$ perovskite lightemitting diodes by utilizing plasmonic Au nanoparticles. J. Phys. Chem. Lett. 8, 3961-3969. doi: 10.1021/acs.jpclett.7b01562

Chen, X., Wu, G., Chen, J., Chen, X., Xie, Z., and Wang, X. (2011). Synthesis of "clean" and well-dispersive Pd nanoparticles with excellent electrocatalytic property on graphene oxide. J. Am. Chem. Soc. 133, 3693-3695. doi: $10.1021 /$ ja110313d

Cho, C., Kang, H., Baek, S. W., Kim, T., Lee, C., and Kim, B. J., et al. (2016). Improved internal quantum efficiency and light-extraction efficiency of organic light-emitting diodes via synergistic doping with $\mathrm{Au}$ and $\mathrm{Ag}$ nanoparticles. ACS Appl. Mater. Interfaces 8, 27911-27919. doi: 10.1021/acsami.6b07666

Choi, H., Ko, S. J., Choi, Y., Joo, P., Kim, T., Lee, B. R., et al. (2013). Versatile surface plasmon resonance of carbon-dot-supported silver nanoparticles in polymer optoelectronic devices. Nat. Photon. 7, 732-738. doi: 10.1038/NPHOTON.2013.181

Coehoorn, R., Eersel, H. V., Bobbert, P., and Janssen, R. (2015). Kinetic monte carlo study of the sensitivity of OLED efficiency and lifetime to materials parameters. Adv. Funct. Mater. 25, 2024-2037. doi: 10.1002/adfm.201402532

Fu, X., Kuang, P., Wang, S., Zhang, G., and Yin, H. (2016). Controllable synthesis of graphene oxide-silver (gold) nanocomposites and their size-dependencies. RSC Adv. 6, 70468-70473. doi: 10.1039/C6RA11291A

Gómez-Navarro, C., Weitz, R. T., Bittner, A. M., Scolari, M., Mews, A., Burghard, M., et al. (2007). Electronic transport properties of individual chemically reduced graphene oxide sheets. Nano Lett. 7, 3499-3503. doi: $10.1021 /$ nl072090c

Han, K. H., Park, Y. S., Cho, D. H., Han, Y., Lee, J., Yu, B., et al. (2018). Optical analysis of power distribution in top-emitting organic light emitting diodes integrated with nanolens array using finite difference time domain. ACS Appl. Mater. Interfaces. 10, 18942-18947. doi: 10.1021/acsami.8b02631

Jesuraj, J. J., Parameshwari, R., Kanthasamy, K., Koch, J., Pfnür, H., and Jeganathan, K. (2017). Hole injection enhancement in organic light emitting devices using plasma treated graphene oxide. Appl. Surf. Sci. 397, 144-151. doi: 10.1016/j.apsusc.2016.11.110

Kim, J. H., Seo, J., Kwon, D. G., Hong, J. A., Hwang, J., Choi, H. K., et al. (2014). Carrier injection efficiencies and energy level alignments of multilayer graphene anodes for organic light emitting diodes with different hole injection layers. Carbon 79, 636-630. doi: 10.1016/j.carbon.2014.08.024

Lee, C. Y., Le, Q. V., Kim, C., and Kim, S. Y. (2015). Use of silane-functionalized graphene oxide in organic photovoltaic cells and organic light-emitting diodes. Phys. Chem. Chem. Phys. 17:9369. doi:10.1039/C5CP00507H

Lee, D. Y., Na, S. I., and Kim, S. S. (2016). Graphene oxide/PEDOT: PSS composite hole transport layer for efficient and stable planar heterojunction perovskite solar cells. Nanoscale 8, 1513-1522. doi:10.1039/C5NR05271H

Li, Y., Li, P., and Lu, Z. (2017). Mapping energy levels for organic heterojunctions. Adv. Mater. 29:1700414. doi: 10.1002/adma.201700414

Liang, J., Li, L., Tong, K., Ren, Z., Hu, W., Niu, X., et al. (2014). Silver nanowire percolation network soldered with graphene oxide at room temperature and its application for fully stretchable polymer light-emitting diodes. ACS Nano. 8 , 1590-1600. doi: $10.1021 / \mathrm{nn} 405887 \mathrm{k}$

Liu, L., Li, S., Zhou, Y., Liu, L., and Cao, X. (2017). High-current stressing of organic light-emitting diodes with different electron-transport materials. Microelectron. Reliab. 71, 106-110. doi: 10.1016/j.microrel.2017. 03.002

Naumov, A., Grote, F., Overgaard, M., Roth, A., Halbig, C. E., Nørgaard, K., et al. (2016). Graphene oxide: a one- versus two-component material. J. Am. Chem. Soc. 138, 11445-11448. doi: 10.1021/jacs.6b05928

Park, M., Nguyen, T. P., Choi, K. S., Park, J., Ozturk, A., and Kim, S. Y. (2017). MoS2 -nanosheet/graphene-oxide composite hole injection layer in organic light-emitting diodes. Electron. Mater. Lett. 13, 344-350. doi: 10.1007/s13391-017-1612-3

Riedel, B., Hauss, J., Geyer, U., Guetlein, J., Lemmer, U., and Gerken, M. (2010). Enhancing outcoupling efficiency of indium-tin-oxide-free organic light-emitting diodes via nanostructured high index layers. Appl. Phys. Lett. 96:243302. doi: 10.1063/1.3455098

Shi, S., Sadhu, V., Moubah, R., Schmerber, G., Bao, Q., and Silva, S. R. (2013). Solution- processable graphene oxide as an efficient hole injection layer for high luminance organic light-emitting diodes. J. Mater. Chem. C 1, 1708-1712. doi: 10.1039/C3TC00707C

Silva, W. J. D., Yusoff, A. R. B., Jang, J. (2013). GO: PEDOT:PSS for high-performance green phosphorescent organic light-emitting diode. IEEE Electron Device L. 34, 1566-1568. doi: 10.1109/LED.2013.22 84272

Sun, Y., and Forrest, S. R. (2008). Enhanced light out-coupling of organic lightemitting devices using embedded low-index grids. Nat. Photon. 2, 483-487. doi: 10.1038/nphoton.2008.132

Wu, X., Li, F., Wu, W., and Guo, T. (2014b). Flexible white phosphorescent organic light emitting diodes based on multilayered graphene/PEDOT: PSS transparent conducting film. Appl. Surf. Sci. 295, 214-218. doi: 10.1016/j.apsusc.2014.01.034

Wu, X., Liu, L., Choy, W. C., Yu, T., Cai, P., Gu, Y., et al. (2014a). Substantial performance improvement in inverted polymer light-emitting diodes via surface plasmon resonance induced electrode quenching control. ACS Appl. Mater. Interfaces 6:11001. doi: 10.1021/am5033764

Wu, X., Zhuang, Y., Feng, Z., Zhou, X., Yang, Y., Liu, L., et al. (2018). Simultaneous red-green-blue electroluminescent enhancement directed by surface plasmonic "far- field" of facile gold nanospheres. Nano Res. 11, 151-162. doi: 10.1007/s12274-017-1614-3

Wu, Y., Zhang, X., Jie, J., Xie, C., Zhang, X., Sun, B., et al. (2013). Graphene transparent conductive electrodes for highly efficient silicon nanostructuresbased hybrid heterojunction solar cells. J. Phys. Chem. C 117, 11968-11976. doi: $10.1021 /$ jp $402529 \mathrm{c}$

Xiao, L., Su, S., Agata, Y., Lan, H., and Kido, J. (2009). Nearly 100\% Internal quantum efficiency in an organic blue-light electro phosphorescent device using a weak electron transporting material with a wide energy gap. Adv. Mater. 21, 1271-1274. doi: 10.1002/adma.200802034

Xiao, Y., Yang, J. P., Cheng, P. P., Zhu, J. J., Xu, Z. Q., Deng, Y. H., et al. (2012). Surface plasmon-enhanced electroluminescence in organic lightemitting diodes incorporating Au nanoparticles. Appl. Phys. Lett. 100:013308. doi: $10.1063 / 1.3675970$

Xu, L., Qiang, Y., Xiao, K., Zhang, Y., Xie, J., Cui, C., et al. (2017). Surface plasmon enhanced luminescence from organic-inorganic hybrid perovskites. Appl. Phys. Lett. 110:233113. doi: 10.1063/1.4985294

Yeo, J. S., Kang, R., Lee, S., Jeon, Y. J., Myoung, N., Lee, C. L., et al. (2015). Highly efficient and stable planar perovskite solar cells with reduced graphene oxide nanosheets as electrode interlayer. Nano Energy 12, 96-104. doi: 10.1016/j.nanoen.2014.12.022

Yun, J. M., Yeo, J. S., Kim, J., Jeong, H. G., Kim, D. Y., and Noh, Y. J. (2011). Solution-processable reduced graphene oxide as a novel alternative to PEDOT:PSS hole transport layers for highly efficient and stable polymer solar cells. Adv. Mater. 23, 4923-4928. doi: 10.1002/adma.201102207

Zhang, C., Marvinney, C. E., Xu, H. Y., Liu, W. Z., Wang, C. L., Zhang, L. X., et al. (2015a). Enhanced waveguide-type ultraviolet electroluminescence from $\mathrm{ZnO} / \mathrm{MgZnO}$ core/shell nanorod array light-emitting diodes via coupling with Ag nanoparticles localized surface plasmons. Nanoscale 7, 1073-1080. doi: 10.1039/C4NR04966G 
Zhang, S., Yao, L., Peng, Q., Li, W., Pan, Y., Xiao, R., et al. (2015b). Achieving a significantly increased efficiency in nondoped pure blue fluorescent OLED: a quasi-equivalent hybridized excited state. Adv. Funct. Mater. 25, 1755-1762. doi: 10.1002/adfm.201404260

Zhang, X., Xu, B., Wang, W., Liu, S., Zheng, Y., Chen, S., et al. (2017). Plasmonic perovskite light-emitting diodes based on the Ag-CsPbBr3 system. ACS Appl. Mater. Interfaces. 9, 4926-4931. doi: 10.1021/acsami.6b12450

Zhu, Y., Murali, S., Cai, W., Li, X., Suk, J. W., Potts, J. R., et al. (2010). Graphene and graphene oxide: synthesis, properties, and applications. Adv. Mater. 22, 3906-3924. doi: 10.1002/adma.2010 01068
Conflict of Interest Statement: The authors declare that the research was conducted in the absence of any commercial or financial relationships that could be construed as a potential conflict of interest.

Copyright (C) 2018 Feng, Sun, Mei, Shi, Mei, Zhou, Xu, Jiang and Wu. This is an open-access article distributed under the terms of the Creative Commons Attribution License (CC BY). The use, distribution or reproduction in other forums is permitted, provided the original author(s) and the copyright owner(s) are credited and that the original publication in this journal is cited, in accordance with accepted academic practice. No use, distribution or reproduction is permitted which does not comply with these terms. 